\title{
Are mothers aware of the importance of infant feeding and paediatric nutrition? A follow-up to the DIT-Coombe Hospital Study
}

\author{
Q. Zhou, K. M. Younger and J. M. Kearney \\ School of Biological Sciences, Dublin Institute of Technology, Kevin Street, Dublin 8, Republic of Ireland
}

Paediatric nutrition, fundamentally influenced by maternal feeding attitudes and behaviours, plays a pivotal role in influencing an individual's health from conception throughout childhood and later during adult life ${ }^{(1)}$. However, current feeding practices do not always concur with the optimal infant feeding recommendations ${ }^{(2)}$. The present project thus seeks to acquire an insight into the maternal infantfeeding attitudes and to identify whether these attitudes differ socio-demographically between subgroups in the population, in order to correctly target and proactively educate mothers in most need of intervention.

A non-randomised postal follow-up survey to the DIT-Coombe Hospital Infant Feeding Study was conducted, involving the recruitment of 209 mothers from a pool sample of 520 mothers in a previous study ${ }^{(3)}$ (participant rate $40.2 \%$, response rate $46.7 \%$ ). Quantitative data were obtained from a cross-sectional questionnaire ranking maternal attitudes towards diet and infant feeding practices on a Likert scale ${ }^{(4)}$ of perceived importance.

\begin{tabular}{lr} 
Table. Percentage of participants who strongly agreed that the following diseases were influenced by infant diet \\
\hline Infant-diet-related diseases & $\%$ of mothers \\
\hline 'Overweight', 'poor growth and development', 'a preference for unhealthy food' & $>85$ \\
'Diabetes', 'osteoporosis', 'high blood pressure', 'heart disease', 'gastrointestinal disease' & $50-85$ \\
'Cancer', 'food allergy', 'eczema, asthma or hay fever', 'mental health problem' & $<50$ \\
\hline
\end{tabular}

A majority of mothers were aware of the long-term health implications of infant diet (Table). However, they did not perceive the paediatric diet to be as strongly influential as the other factors (physical activities in adulthood and childhood, smoking and diet in adulthood) on adult health. Of the mothers $<30 \%$ considered breast-feeding and weaning age to be very important to adult health. There was also a lack of knowledge about the protective effect of breast-feeding against infection, with less than half the samples agreeing that breast-feeding results in fewer infections than formula feeding. Univariate analysis indicated that mothers who had initiated breast-feeding after birth and who had a higher education level were more likely to be aware of the advantages of breast-feeding and appreciate weaning $(P<0.05)$. Mother's age at the time of birth, marital status, social class and their partner's social class were also predictors of maternal attitudes toward infant feeding practices, but not as strong as education level or initiation of breast-feeding.

Although there is wide awareness of the health impacts of paediatric nutrition, there is still a need to stress the importance of breastfeeding and appropriate weaning to the mothers, and correct some misconceptions, in order to promote the optimal infant feeding practices. Those mothers who are less educated could be selectively targeted.

1. Barker DJP, Erikson JG, Forsen T \& Osmond C (2002) Int J Epidemiol 31, 1235-1239.

2. Cattaneo A, Yngve A, Koletzko B \& Guzman LR (2005) Public Health Nutr 8, 39-46.

3. Tarrant RC, Younger K, Sheridan-Periera M \& Kearney JM (2007) Proc Nutr Soc 66, 54A.

4. Nayak L \& Erinjeri JP (2008) Acad Radiol 15, 383-389. 